\title{
Psychological safety as a characteristic feature of common education environment of higher educational institutions in times of globalization
}

\author{
Nadezhda Nichiporenko*, Anna Zhemchugova \\ Federal State Budget Scientific Institution «Institute of Pedagogy, Psychology and Social Problems», \\ Laboratory of Reseach of Deviant Behavior, 420039, Isaev str, 12, Kazan, Russia
}

\begin{abstract}
The article is devoted to the analysis of the results of monitoring carried out in higher educational institutions of the Republic of Tatarstan (more than 30 universities, institutes and their regional subdivisions). 47433 students were surveyed for the present analysis. The goal of the research was to study the parameters of educational environment that might potentially influence on individual's psychological need for security. During the monitoring the students' depression and aggression levels were measured. Questionnaire touched upon psychological security problems, such as relationships with teaching staff, relationships with peers, situations of psychological abuse (bullying and mobbing), emotional attitude towards studying. According to correlation analysis that was carried out, it was possible to single out multiple cases of correlation between aggression and depression with various security components of educational environment. The article reflects the fact that depression and aggression represent not only generalized, obscuredly specific patterns of psychological ill-being of a certain person, but they are known to be adjustment mechanisms that help to cope with adverse circumstances. Being closely related to different factors of schooling, depression and aggression can serve as secure and valid subjective markers of psychological security of a certain person in the educational environment.
\end{abstract}

\section{Introduction}

Rapid changes of modern educational process under the conditions of transformation to information-oriented society is a direct result of international processes of globalization and digitalization. Any innovations and transformations in social reality are inevitably connected to certain risks and can lead to a decrease in psychological safety of personality. Nowadays this tendency is also intensified by such factors as political and economic instability, threat to life and health in times of pandemic, toxic mass media. Yu.P. Zinchenko addresses some problems of safety in broader sense, defining this parameter as the activity of certain social subjects and relations between them as a sphere where social, collective and individual consciousness can function and develop [1]

\footnotetext{
${ }^{*}$ Corresponding author: nichiporenko.n@yandex.ru
} 
Management of common education environment even in one particular state can be extremely challenging as it is. In case we need to talk about creation of educational environment, that has to integrate various mental and cultural patterns of Europe, Russia and China, it is important to sustain unique character and identity of the subjects of educational environment on the one hand and to define common features and parameters that might be universal and weakly specific (free from the cultural influence) on the other hand. Therefore, psychological safety of education environment is in fact a priority in terms of universal human values and thus deserves a status of independent topic for cross-cultural research.

The foundation for studying different characteristics of environment as a factor for personality development were reflected in cultural and historic theory of L.S. Vygotsky and reflected in the definition of "social situation of development". According to L.S. Vygotsky social situation of development corresponds to "unique, specific for a certain age, exceptional, one and only relation between a child and surrounding environment (mostly social environment)" [2]. The definition of "educational environment" appeared and was developed later on. Ecological and person-centred model of the environment includes scope of society, organizational structure of society, as well as culture, technogenic sphere and it is assumed that interaction of different components lies foundation for this environment [3]. G.A. Kovalev described three-component structure of educational environment. This includes "human factor", "physical surrounding" and "curriculum" [4]. This structure became a benchmark for a further structural and content-related analysis of the environment in many different psychological research works. V.V. Rubtsov brought to the foreground psychological characteristics of the environment and defined education environment as a form of communicative interaction. He considered the components of the education environment to be psychological organization of knowledge transmission, psychological climate and social and psychological structure of a team as well as psychological characteristics of the students [5].

Hereafter the researchers shift the focus onto subject and object relations of a person and the environment. V. I. Slobodchikov mentions adaptability of educational environment as its ability to assimilation and accommodation [6]. From the point of view of subjects of educational environment its adaptability is defined by "possibilities" that this environment provides to satisfy individual needs. These "possibilities" become the mediators between subject and environment. "Possibilities" here are defined both as features of environment and as features of subject. Thus the experience of environment by subject starts to play an important role. In this respect, impersonal space becomes an environment only in case when conditions become possibilities. Discrepancies between these two sides of interaction (conditions and possibilities) can lead to a certain psychological ill-being, disturbance of an emotional balance and even to increased level of aggression of the subjects of environment [7].

For modern research of educational environment safety discourse is of immediate interest. I.A. Baeva defines psychological safety of environment as "a condition of the environment free from manifestations of psychological abuse during the interaction; it also promotes satisfaction of the needs in personal open communication and creates benchmark significance of the environment and provides psychological well-being of the members that are involved in it" [8]. Markers of well-being of educational environment might be emotional and personal as well as communicative characteristics of its subjects, such as self-actualization, self-attitude, commitment to communication, level of self-centering and centering on others, low level of emotional tension [9]. Safety risks of school environment are represented by violation of subjective well-being, discipline, environmental comfort, as well as expressions of violence, substance abuse, cross-ethnical conflicts, suicidal behavior, psychological traumas of different origins [10]. 
The research of the factors that reflect tension in the educational environment in higher educational institutions shows that safety of educational environment can be determined by the results of both external and internal factors and influences. In this respect any pressure, including the pressure from the environment can lead to two oppositely directed results: either external resistance, such as aggression, breaking of customary norms, or internal, when the response to pressure is social withdrawal, avoidance of the contact and autoaggressive tendencies [11].

In foreign countries the research works on depressive tendencies in students are widely spread, because depression is considered to be main psychological disorder, that many students face during their university years. Prevalence of depressions among students might be connected to sudden changes in the conditions of their lives when they enter the university, or appearance of other more severe psychological, social and economic demands from the society [12].

From the perspective of the present research, the work of A. Fung, L. Gerstein, Y. Chan and J. Engebretson [13] might be of a certain value. They have established the connection between aggressive manifestations and depressive conditions in students. It is important to mention that the degree and character of severity of aggressive tendencies among students that were previously diagnosed with depression and that clearly demonstrate depressive patterns of the behavior, are connected with the structure of depression and type of dominant affect. For instance, it was shown that in the group of students that had a combination of symptoms of depression and high levels of hostility and aggression, the type of dominant affect was anxiety. It is increased anxiety in the structure of depression that leads to manifestations of both indirect and direct verbal and physical aggression. This becomes particularly evident in the form of irritation, fears and instability of emotional processes. At the same time the students, who had despair as their dominant affect in the structure of depression, were less inclined to demonstrate aggressive and hostile patterns of the behavior. Signs of depressive state are mostly of passive character. The other research demonstrated the evidence of the fact that the level of aggression can increase in the presence of low level of intellectual abilities and inability to interpret personal emotions and conditions [14].

While studying clinical and patho-genetic aspects of depression typology, the staff members of Moscow Psychiatric Research Institute proved that the affective component of depressive syndrome (anxiety, despair, apathy) define structural peculiarities of depressive state, as well as direction and level of aggression and the degree of violation of social and psychiatric functioning [15]. Earlier in her works A.V. Vaxman paid attention to the fact that high level of aggression combined with depressive episode leads to increase in the degree of suicidal risks, as well as risks of hostile anti-social behavior and misanthropy. In case the level of aggression is low, suicidal behavior is usually of demonstrative character, however the somatic component of the depression might be clearly explicit (vegetative disorders, cardiovascular and respiratory symptoms) [16].

Generally, a combination of depressive and aggressive patterns is clearly visible in younger patients, as well as emotionally unstable patients, that show tendency to displaying demonstrative and narcissistic behavior. Males tend to demonstrate this type of combination more than females [17].

The combination of such factors as aggression and depression has a direct correlation with social disadaptation in young adults and violations of interpersonal interaction in all spheres. It is worth mentioning that depressive patients with low level of aggression might have deterioration mostly in studying and work-related spheres, connected with decrease in cognitive abilities, which is common in cases of depression.

Thus, favorable and safe educational environment forms stable personality that might be resistant to external influences. Signs of disadaptation and ill-being are such symptoms as 
high level of aggressive and depressive tendencies among the subjects of the environment. The aim of the present research work is the study of characteristics of psychological safety of educational environment in higher educational institutions.

\section{Methods and research methodology}

The Republic of Tatarstan is one of the biggest regions with quite a wide spectrum of ethnical, cultural and confessional diversity. It is a unique region of the Russian Federation, that manages to successfully integrate eastern and western traditions. Due to these characteristics and favorable geographical location, Tatarstan can serve as a multicultural model and platform for implementing cross-cultural research.

47433 students were surveyed for the present analysis - they are the students of more than 30 educational institutions and their regional subdivisions, 45578 of them are undergraduate students, 1855 - students doing Masters courses, 19238 are females, 28195 are males.

In the process of monitoring levels of students' depression and aggression were surveyed by means of testing as well as filling in the questionnaires. A complete version of the questionnaire consisted of 33 questions, that touched upon different aspects of relations between the subjects of the educational environment. Present article analyzes certain selected questions that were given in the questionnaire. These questions are directly related to problems of psychological security, such as relationships with teaching staff, relationships with peers, situations of psychological abuse, emotional attitude towards studying.

Psychodiagnostic research methodology. 1. The Zung self-rating depression scale (adaptation made by T.I. Balashova in the Addictology Department of Research Institute named after Bekhterev). This questionnaire was developed for the purposes of differential diagnostic procedure of depressive and near-depressive conditions. It is effective for screening in mass research as well as for the purposes of premedical diagnostics. 2. BPAQ24 (Buss-Perry Aggression Questionnaire) developed by A. Buss and M. Perry for the purposes of diagnostics of inclination to aggression and of three aggression factors: physical aggression, anger and hostility. Adaptation of this methodology in Russia was carried out by the experts of the laboratory of clinical psychology in Russian Academy of Medical Sciences S.N. Enikolopov and N.P. Tsibulskiy. They adapted the questionnaire by testing law-abiding trial subjects and criminals that were sentenced for committing aggressive and violent crimes (murder, assault, rape abuse, causing a dreadful injury, gross misconduct). The results of adaptation and standardization of the present methodology on the contrasting groups of trial subjects proved high level of validity and reliability of the Russian version of the questionnaire (BPAQ-24) and its feasibility for the purposes of diagnostics of physical aggression inclinations, as well as hostility and anger. Specified three-factor structure of the questionnaire corresponds to theoretical disposition of three components of aggression in the concept of A. Buss and M. Perry: instrumental (physical aggression), emotional (including physiological excitation and affective preparation to aggression - anger) and cognitive (cognition of injustice and infringement, unfulfilled desires - hostility).

\section{Discussion and analysis of the results}

In order to define the correlation between the studied parameters, the correlation analysis (Pearson method) was used.

The correlation between depression and aggression is direct and statistically evident with the high level of significance $(r=0.547, \mathrm{p}<0.001)$. 
The questions of the questionnaire were grouped according to the content, depending on the aspect of relations they touch upon.

The first group - "Emotional attitude towards studying" - included 6 questions, relating to positive emotions in the process of studying (interest, joy, satisfaction), academic overload, possibility to regulate the level of self-participation in the social events, possibility to openly show your point of view, as well as some questions related to negative experience in relation to studying, teaching staff and fellow students - fear, anger, grievance, accusations. All correlations between depression/aggression and positive emotional states are negative $(-0.37 \leq \mathrm{r} \leq-0.21, \mathrm{p}<0,001)$, and correlations with negative emotions are positive (connection with depression $\mathrm{r}=0.33, \mathrm{p}<0.001$, with aggression $\mathrm{r}=0.34$, $\mathrm{p}<0.001)$.

The second group - "Relationship with teaching staff" - consisted of 3 questions that were aimed at identifying the level of security from unsubstantiated criticism from teachers (connection with depression $\mathrm{r}=-0.32, \mathrm{p}<0.001$, with aggression $\mathrm{r}=-0.32, \mathrm{p}<0.001$ ), unrighteous accusation (connection with depression $\mathrm{r}=-0.25, \mathrm{p}<0.001$, with aggression $\mathrm{r}=$ $0.25, \mathrm{p}<0.001$ ), capability to receive support and help from teachers (connection with depression $r=-0.35, p<0.001$, with aggression $r=-0.34, p<0.001$ ).

The third group consisted of 6 questions that were aimed at identifying various aspects of relationships with fellow students. The following things were tested: support and help from peers in the process of problem solving (connection with depression $r=-0.32$, $\mathrm{p}<0.001$, with aggression $\mathrm{r}=-0.32, \mathrm{p}<0.001$ ), trust (connection with depression $\mathrm{r}=-0.32$, $\mathrm{p}<0.001$, with aggression $\mathrm{r}=-0.38, \mathrm{p}<0.001$ ), mutual help (connection with depression $\mathrm{r}=-$ $0.30, p<0.001$, with aggression $r=-0.37, p<0.001$ ), absence of hostility (connection with depression $\mathrm{r}=-0.20, \mathrm{p}<0.001$, with aggression $\mathrm{r}=-0.30, \mathrm{p}<0.001$ ), presence of friends (connection with depression $\mathrm{r}=-0.35, \mathrm{p}<0.001$, with aggression $\mathrm{r}=-0.29, \mathrm{p}<0.001$ ), opportunity to share joy or problems (connection with depression $r=-0.36, p<0.001$, with aggression $r=-0.29, p<0.001$ ), security among peers (connection with depression $r=-0.33$, $\mathrm{p}<0.001$, with aggression $\mathrm{r}=-0.31, \mathrm{p}<0.001)$.

Two questions from the questionnaire were directly related to bullying and mobbing. $12,9 \%$ of the respondents had an experience of being bullied in their educational institutions (schools and universities), while 16,9\% of respondents witnessed this phenomena. Correlations with depression for those, who were the victims of bullying $r=0.31$, for those who witnessed that $r=0.22(p<0.001)$. Correlation with aggression $r=0.33$ and $\mathrm{r}=0.31$ respectively $(\mathrm{p}<0.001)$.

While analyzing the results of the research we are going to use several connotations of the term "aggression". Aggression is stable characteristics of the subjects that reflects their predisposition towards the behavior that is aimed at harming surroundings. Aggression is also defined as psychological condition, complex of affects, including such emotions as irritation, anger, rage, grievance, wrath and hatred. Aggression in its broader meaning is state of activeness that is aimed at achieving the goals, overcoming the obstacles. Constructive aggressive behavior is one of the forms of reacting to different unfavourable (both physical and psychological) factors that trigger deprivation of certain important needs, stress and frustration. Destructive aggression is a type of motivated behavior that brings harm to inanimate and animate objects of attacks, bringing physical, moral harm to people or triggering a certain psychological discomfort.

In the present research the term "depression" also has several connotations. Depression can have a status of stable personal characteristics, that is represented in the form of atonic state of need and motivation, inclination to perceive and interpret the events in a negative way, low level of vital energy, apathy and passiveness. The notion of depression can also describe an emotional phenomenon (psychological state) with prevailing decreased mood, melancholy, despair, asthenia, helplessness, frustration. The term "depression" can also 
characterize a wide spectrum of premorbid and clinically apparent conditions ranging from subdepressive manifestations to profound forms of endogenous depression.

Aggression and depression represent certain personal characteristics that might be clearly measured, moreover they have a high level of prognostic value in the sphere of prediction of certain developmental deviations. It is important to single out the fact that both aggression and depression are to some extent generalized, weakly specific psychological phenomena that might trigger or be a cause of forming certain abnormalities of different spectrum, such as mental health conditions and personality disorder, psychosomatic reactions, conditions and illnesses, varied forms of social alienation, delinquent behavior, criminal activity. Being to some extent closely connected to such a varied spectrum of deviations, aggression and depression are used in the present research as markers of psychological ill-being.

Prepubescent years and adolescence represent periods of intensive personal growth, it is the time of self-identification and personalization. During the university years students continue to go through separation processes, as well as identity forming processes. It is possible to say that to a certain extent they confront multiple socialization challenges that society places in front of them. It is possible to say that conflict of individuation and socialization processes reaches its peak in adolescence compared to other developmental periods. Age-specific tasks of this developmental stage are complex and controversial, while there are usually not enough skills to cope with them, as ways of coping with difficult life situations are still in the process of formation. From this point of view students of higher educational institutions represent on the one hand a certain risk group when it comes to reinforcement of negative scenarios of their life journey and on the other hand a wide target audience for measures that might be taken in order to prevent different deviations in the older age.

Aggression in adolescence and prepubescent years is not necessarily a sign of negative trait of character. In certain cases the absence of aggression in this period may cause more worries than openly aggressive behavior. Separation processes form personal boundaries, determinate the privacy of the psychological space of personality and as a result it is impossible to carry out these tasks without a certain level of aggression. The development of identity is carried out with the help of two different mechanisms - identification with a certain social group and differentiation of "your own" social group from the community of "others". This causes an underlying conflict, and in certain cases even confrontation, when you have to differentiate "yours" from "others", "yourself" from "stranger" and sometimes you have to be able to stand your ground in a conflict with "others". In separation process and personality forming process aggression plays an instrumental role, it serves the tasks of adaptation and has a clearly constructive character. That's why we claim that insignificant increase of psychological and social norms of aggressiveness in adolescence and prepubescent years is quite acceptable and it is usually a feature of normal stage in development process of this age rather than a sign of deviation or personality disorder. Abnormal variations of the development are represented by two types of destructive aggression. Firstly, it is autoagression and its extreme manifestation, i.e. suicidal behavior. Secondly, it is hetero-aggressive behavior, in case the forms of this type of aggression assume a form of delinquency, asocial, antisocial or criminal activity.

The main sign that might differentiate a "normal" type of aggression is the fact that the person is able to regulate his aggressive behavior and his own level of aggression. Thus the criterion of the norm of aggression is not just its level, but rather presence of selfregulation.

Depression as well as aggression can fulfil certain adaptive functions. Depressive states decrease general level of functioning, decline the intensiveness of external activity, save strength and allow to withstand in the times of hardship. Depressive tendencies are to 
certain extent an opposition to aggressive tendencies, and thus they are effective in the circumstances when it is useless to use aggression. These are the situations when active actions can cause a profound damage to the personality itself, while inertness can be a good solution. In terms of emotions these situations are usually connected with such feelings as disappointment, despair, asthenia, helplessness, frustration. In case these circumstances have a prolonged character and become chronic, depressive patterns of coping can become settled. Long-term usage and aggravation of depressive ways of reacting might increase the risk of psychological disorders, promote personality disorder, intensify autoaggressive tendencies and promote suicide.

Psychological safety of the educational environment is a multifactorial phenomenon, that might be considered as a combination of external conditions of studying and their internal representations. The questions in the questionnaire, that were relevant to the research topic, were divided into 4 groups and thus it became possible to analyze fourcomponent structure of psychological safety of educational environment. These components are emotional attitude towards studying, relationships with teaching staff, experience of psychological abuse, relationships with peers (other students, groupmates).

Emotional attitude towards studying process is expressed via positive emotions such as interest, joy, satisfaction, which in turn indicate that the atmosphere in the university (institute) is perceived as being comfortable and safe. The spectrum of these emotions is connected to aggression and depression reciprocally. Generally, it is considered to be a reliable subjective indicator of psychological safety of the students. Fear, anger, grievance and unsubstantiated accusations from the teachers have a direct correlation with aggression and depression in students. From the point of view of emotional comfort, it is essential to give students opportunity to self-regulate the level of their involvement in the public events, and it is also important for them to be able to express their point of view freely. Moreover, the university administrative staff and the studying schedule itself should allow students to regulate their academic workload in order to avoid exhaustion. All these factors create supportive environment for the development of metacognitive studying skills, such as goalsetting, monitoring, planning, self-regulation and self-control. It might be problematic for the $1^{\text {st }}$ year students, because these skills are usually still underdeveloped due to the fact that school education system uses mostly external control and external motivation. As a result, this hinders the development of self-organization and self-regulation. The opportunity to regulate academic overload, avoid exhaustion and not to take part in the events, that are considered to be less important by the students themselves are important for psychological safety as this creates internal feeling of control and possibility to influence the circumstances, regulate the environment, plan their time and resources.

Relationship with teaching staff is also an important component of the educational environment, that influences the perception of psychological safety of the students. Being protected from unfair criticism and unsubstantiated accusations from the teachers, opportunity to receive support and help from the teaching staff not only helps to minimize the expectancy of external danger, but also becomes the factor of preventative measures against such feeling as hopelessness. It should be taken into consideration that during the first years of studying at university students are still really sensitive towards criticism and assessment from third parties. The feelings of guilt and shame (especially narcissistic shame) represent very strong toxic emotional experience, that directly jeopardizes both selfrespect and self-esteem and at times even the integrity. It looks like shame and guilt, that were induced by significant grown-ups (parents, teachers), are the most important ways of influencing the developing personality. These feelings represent main parameters of psychological unsafety in modern educational environments (these might be school, university or even family). 
Relationship with peers as a factor of psychological safety was represented in the questionnaire by such parameters as feeling of security among peers, support and help from groupmates in problem solving, trust and mutual help, absence of hostility, presence of friends, possibility to share joy or problems. All these characteristics of social contacts have negative correlations with aggression and depression and thus can definitely be considered as important factors that reflect safety of educational environment.

Bullying and mobbing are quite wide-spread ways of stigmatizing in different groups of people. However, these two phenomena represent extremely dangerous forms of psychological abuse specifically among adolescents and young adults in different educational institutions, because they trigger traumatizing of both the victim and the witnesses of these phenomena. The main components of psychological trauma in this case are asthenia (inability to stand against the collective aggressor) and helplessness (inability to turn for help neither to teaching staff, nor to groupmates; very often the victims of bullying refuse to talk about their negative experience even with their parents). There are three specific age-related factors that can multiply the traumatic experience. The first one is high importance (referentiality) of relationships with peers. The second factor is imperfection of well-established psychological defenses and lack of efficient coping mechanisms. The third factor is desperation, when the students feel that it is impossible to solve a situation or make a safe getaway. Not everyone is eager to change the educational institution or class, and at the same time parents usually underestimate the level of real danger, while prestige, intellectual status of school, institute or university become a real priority as opposed to psychological safety of the child. There is a clear correlation between the experience of bullying or facing this as a witness and both depression and aggression. It should also be mentioned that coefficients of correlation for witnesses and victims are very close for the parameter of "depression" ( 0.31 and 0.22 correspondingly) and are almost identical for the parameter "aggression" $(0.33$ and 0.31$)$. Thus it is possible to conclude that any type of involvement in abuse in educational environment represents a real threat to psychological safety of all subjects of educational cooperation.

\section{Conclusions}

1. Depression and aggression can serve as secure and valid subjective markers of psychological security of a certain person in the educational environment.

2. Any type of involvement in abuse in educational environment represents a real threat to psychological safety of all subjects of educational cooperation.

3. The important parameters of safe educational environment in academic institution of higher education are relationships with peers (other students), relationships with teaching staff and common emotional context of the educational institution.

4. Depression and aggression represent not only generalized weakly specific markers of psychological ill-being of personality, but are also mechanisms of adaptation to unfavourable circumstances.

\section{Acknowledgements}

The research was carried out by state order №0599-2019-0043 "Problem of deviant behavior in the system of modern study of human nature".

\section{References}

1. Yu.P. Zinchenko, National Psychological Journal, 2(6), (2011)

2. L.S. Vygotsky, Age problem, (1984) 
3. O.D. Dunkan, L.F. Shnore, Amer. Journal of Sociology, 65, (1969)

4. G.A. Kovalev, Voprosy Psychol., 1, (1993)

5. V.V. Rubtsov, Second Rus. conf. on environmental psychology, (2000)

6. V.I. Slobodchikov, Second Rus. conf. on environmental psychology, (2000)

7. V.A. Yasvin, Journal "Izvestiya of Saratov University. Series: Philosophy. Psychology. Pedagogy", 18(1), (2018)

8. I.A. Baeva, E.V. Burmistrova, E.B. Laktionova, N.G. Rassokha, Ensuring psychological safety in an educational institution, (2006)

9. I.A. Baeva, L.A. Gayazova, Psychological Science and Education, 4(3), (2012)

10. I.A. Baeva, Kazan pedagogical journal, 125(6), (2017)

11. M.I. Bekoeva. Baltic humanitarian journal, 4(21), (2017)

12. A. Hamdan-Mansour, J. Halabi, \& H. Dawani, International Journal Mental Health Addiction, 2(1), (2008)

13. A. Fung, L. Gerstein, Y. Chan, \& J. Engebretson, Journal Child Family Studies, 24, (2015).

14. P Rey, L. \& N. Extremera, Universitas Psychologica, 11(4), (2012)

15. O.P Vetrogradova., I.L Stepanov., N.M Maximova., A.V Vaxman., S.Yu Dikov., K.A Koshkin., O.V.Moiseycheva, O.V. Tselischev, Social and clinical psychiatry, 22(3), (2012)

16. A.V. Vaxman, Aggression and socio-mental functioning of patients with depression, (2004)

17. L. Burton, J. Hafetz \& D. Henninger, Social Behavior \& Personality, 35(1), (2007) 